\title{
Prevention and Treatment of Ovarian Hyperstimulation Syndrome
}

\author{
Ivan Grbavac, Dejan Ljiljak and Krunoslav Kuna \\ University Hospital Center "Sisters of Mercy", Zagreb
}

Croatia

\section{Introduction}

Ovarian hyperstimulation syndrome (OHSS) is a clinical symptom complex associated with ovarian enlargement resulting from exogenous gonadotropin therapy. In severe cases, a critical condition develops with massive ascites, marked ovarian enlargement, pleural effusion, electrolyte imbalance, hypovolemia with hypotension, oliguria, hemoconcentration, and thromboembolism (Madill JJ et al., 2008). Moderate to severe ovarian hyperstimulation syndrome (OHSS) has been calculated to occur in $0.2 \%$ to $2 \%$ of all ovarian stimulation cycles (Binder $\mathrm{H}$ et al., 2007).

Severe OHSS can be a life-threatening complication. The prognosis is usually worse in patients who get pregnant and have this syndrome.

\section{Pathophysiology}

The cause of OHSS is unknown, but it may be mediated by vasoactive cytokines secreted in excess by hyperstimulated ovaries (Goldsman MP et al., 1995). Human chorionic gonadotropin (hCG), either exogenous or endogenous (derived from a resulting pregnancy), is believed to be an early contributing factor. Increased vascular permeability is believed to result from vasoactive substances produced by the corpus luteum in response to hCG stimulation (Chen SU et al., 2010).

The pathophysiology of OHSS is characterized by increased vascular permeability with loss of fluid, protein, and electrolytes into the peritoneal cavity (Tollan A et al., 1990).

Gastrointestinal system findings include ascites (third-spacing of fluid), paralytic ileus, and enlarged ovaries. Pulmonary system findings include pleural effusions, restrictive lung disease from ascites or paralytic ileus, and ARDS. Cardiovascular system findings include decreased intravascular volume, decreased blood pressure, decreased central venous perfusion, and compensatory increased heart rate and cardiac output with arterial vasodilation. Coagulation abnormalities include hemoconcentration, increased estrogen level leading to hypercoagulability, and thrombosis. Renal system findings include decreased renal perfusion with subsequent oliguria or renal failure. Hepatic system findings may include hepatic edema. 
Hematologic findings include increased hematocrit (secondary to increased capillary permeability and fluid loss) and elevated white blood cell count, a multifactorial finding associated with elevated estrogen level, prostaglandins, and dilution.

Gynecologic findings include enlarged ovaries which may torse or rupture. Finally, electrolyte findings classically include hyponatremia (secondary to increased antidiuretic hormone due to decreased intravascular volume) and hyperkalemia (secondary to the renal sodium/potassium pump alterations).

\section{Hemodynamic changes}

The clinical manifestations originate from the combination of decreased intravascular space and the accumulation of protein-rich fluid into body cavities and interstitial space. Loss of intravascular volume leads to hemodynamic changes manifested as hypotension, severe tachycardia, and decreased renal perfusion as well as hemoconcentration.

Because hypotension leads to decreased venous pressure and reduced venous return, a decreased cardiac output (CO) might be expected; however, it was found the $\mathrm{CO}$ was increased in OHSS. These findings led to the determination that there is accompanying arterial vasodilation in OHSS (Manau D et al., 1998).

Decreased renal perfusion leads to a decreased glomerular filtration rate (GFR), and can result in oliguria. Changes in perfusion can affect liver function, including synthesis of proteins, of which anticlotting factors are among the first to become depleted (Fabreuges F et al., 1999).

Hemoconcentration with increase in blood coagulability is responsible for arterial and venous thrombotic phenomena in patients with OHSS (Chan WS et al., 2009).

Loss of intravascular volume combined with decreased renal perfusion results in electrolyte abnormalities (hyperkalemia, hyponatremia), increase in hematocrit and white cell count, and decrease in creatinine clearance.

Abdominal discomfort is the most common symptom of OHSS because of the development of ascites. Accumulation of protein-rich fluid in the peritoneal cavity leads to abdominal distention and increased intra-abdominal pressure (IAP). Increased IAP may compromise respiratory, cardiovascular, renal, gastrointestinal and hepatic system homeostasis (Selgas R et al., 2010).

More reccently, vascular endothelial growth factor (VEGF), has emerged as one of the factors most likely to be involved in the patophysiology of OHSS. VEGF is a vasoactive glycoprotein (cytokine) which stimulates endothelial cell proliferation, cell permeability, and angiogenesis.

Recent studies indicate that hCG is the main factor that triggers OHSS and seems to be the main stimulus of the syndrome because elimination of hCG will prevent the full-blown picture of the syndrome (Soares SR et al., 2008). It has been clearly demonstrated that hCG increases VEGF and its VEGF-2 receptors (VEGFR-2) in human granulosa-lutein cells and raises serum VEGF concentration.

VEGF causes an increase in vascular permeability by rearranging endothelial junction proteins, including cadherin and claudin 5. When evaluating human endothelial cells from 
umbilical veins (used as an in vitro model of OHSS), hCG and VEGF caused changes in the actin fibers that are indicative of increased capillary permeability, and cadherin concentration was elevated when hCG and VEGF were added, but not with the addition of estradiol (Villasante A et al., 2007). Cadherin is a soluble cell adhesion molecule that may play a key role in the pathophysiology and progression of vascular hyperpermeability (Villasante A et al., 2007).

\section{Classification of OHSS}

Conventional OHSS staging has relied on clinical symptoms and laboratory findings to categorize severity of disease. The most popular classification system for staging OHSS is that of Golan et al., 1989. This system incorporated the use of transvaginal sonography for both estimating of ovarian enlargement and detection of ascites. The detection of ascites establishes the diagnosis of moderate OHSS which may deteriorate to a severe form and is therefore of major importance. Subsequent modifications defined a group of critical OHSS and added to the severe category of the syndrome (Jenkins JM et al., 2006).

\begin{tabular}{|l|l|}
\hline \multicolumn{1}{|c|}{ Grade } & \multicolumn{1}{c|}{ Symptoms } \\
\hline \multirow{4}{*}{ Mild OHSS } & $\begin{array}{l}\text { Abdominal bloating } \\
\text { Mild abdominal pain } \\
\text { Ovarian size usually }<8 \mathrm{~cm}^{*}\end{array}$ \\
\hline Moderate OHSS & $\begin{array}{l}\text { Nausea }+/ \text { - vomiting } \\
\text { Ultrasound evidence of ascites } \\
\text { Ovarian size usually } 8-12 \mathrm{~cm}^{*}\end{array}$ \\
\hline \multirow{5}{*}{ Severe OHSS } & $\begin{array}{l}\text { Clinical ascites (occasionally hydrothorax) } \\
\text { Oliguria } \\
\text { Haemoconcentracion hematocrit }>45 \% \\
\text { Hypoproteinaemia } \\
\text { Ovarian size usually }>12 \mathrm{~cm} *\end{array}$ \\
\hline \multirow{3}{*}{ Critical OHSS } & $\begin{array}{l}\text { Tense ascites or large hydrothorax } \\
\text { Haematocrit }>55 \% \\
\text { Whitw cell count }>25000 / \mathrm{ml} \\
\text { Oligo/anuria } \\
\text { Tromboembolism } \\
\text { Acute respiratory distress syndrome (ARDS) }\end{array}$ \\
\hline
\end{tabular}

*Ovarian size may not correlate with severity of OHSS in cases of assisted reproduction because of the effect of follicular aspiration

\section{Table 1. Classification of severity of OHSS}

Depending on the time of onset, a division of OHSS into 'early' and 'late' may be useful in determining the prognosis. OHSS presenting within 9 days after the ovulatory dose of hCG is likely to reflect excessive ovarian response and the precipitating effect of exogenous hCG administered for final follicular maturation. OHSS presenting after this period reflects endogenous hCG stimulation from an early pregnancy. Late OHSS is more likely to be severe and to last longer than early OHSS (Mathur RS et al., 2000). 


\section{Risk factors}

The following factors increase the risk independently for the development of OHSS (Enskog A. et al., 1999):

- $\quad$ young age

- low body mass index (BMI)

- $\quad$ polycystic ovarian syndrome (PCOS)

- allergic history

- $\quad$ high antral follicle count

- high doses of gonadotropins

- $\quad$ high or rapidly rising estradiol levels

- large numbers of large and medium-sized follicles

- large numbers of oocytes retrieved

- $\quad$ high or repeated doses of hCG

- pregnancy

- $\quad$ prior OHSS

High or rapidly rising estradiol levels are particularly unreliable and over-rated predictors of OHSS (Papanikolau EG et al., 2006).

An estradiol cut-off of 3,000 pg/mL will miss $2 / 3$ of patients with severe OHSS. Counting the number of $12 \mathrm{~mm}$ follicles is actually a better predictor of OHSS than serum estradiol levels, and the combination of an estradiol level of 5,000 pg/mL and eighteen $12 \mathrm{~mm}$ follicles was found to be the best predictor of OHSS in this study, yielding a sensitivity of $83 \%$ and specificity of 84 \%(Papanikolau EG et al., 2006).

\section{Prevention}

The keys to preventing OHSS are experience with ovulation induction therapy and recognition of risk factors for OHSS.

Caution is indicated when any of the following indicators for increased risk of OHSS are present (Practice Committee of American Society for Reproductive Medicine, 2008):

- $\quad$ rapidly rising serum $\mathrm{E}_{2}$ levels

- $E_{2}$ concentration in excess of $2500 \mathrm{pg} / \mathrm{ml}$

- $\quad$ the emergence of a large number of intermediate sized follicles (10-14mm)

- OHSS prevention has historically included the following strategies:

\subsection{Cycle cancellation}

IVF cycle cancellation with withholding of hCG trigger is the most effective preventative technique, but is emotionally and financially stressful for all involved. Cycle cancellation is generally reserved for patients with a history of severe OHSS in a prior cycle and in cases of total loss of control of the cycle.

\subsection{Coasting}

Coasting involves temporarily stopping gonadotropin administration and postponing the hCG trigger until the estradiol $\left(E_{2}\right)$ level is lower. The proposed mechanism of coasting is as 
follows: lower gonadotropin stimulation leads to decreased $\mathrm{LH}$ receptors, leading to decreased luteinization, and subsequent decreased VEGF levels. Lower gonadotropin stimulation may also increase the rate of granulose cell apoptosis, especially of smaller follicles. Coasting lowers the level of follicular fluid VEGF, thereby potentially preventing the development of OHSS (Tozer AJ et al., 2004).

It is a good alternative that can be used to avoid cycle cancellation in extremely high responders to ovulation induction, who have high risk of developing severe OHSS. Even if OHSS develops after coasting, both incidence and severity will be diminished. However, prolonged coasting has a drawback of a reduced pregnancy rate (GarciaVelasco JA et al., 2006).

\subsection{Decreasing the dose of hCG trigger}

Although theoretically it makes sense to reduce the dose of hCG, there is little data to support this practice and studies are either limited by small sample size or not powered to detect a difference.

\subsection{Agonist trigger}

Using an agonist medication to trigger ovulation has been proposed as another strategy to prevent OHSS. Agonist trigger can only be used in the setting of an antagonist protocol.

Comparing pregnancy rate per patient randomized to GnRH agonist vs. hCG trigger identified no difference in number of oocytes retrieved, fertilization rate, or embryo score.

No patients randomized to either GnRH agonist or hCG trigger developed OHSS; but there was the suggestion of a lower pregnancy rate in patients who had GnRH agonist trigger, possibly due to a luteal support problem (Griesinger G et al, 2006).

\subsection{Cryopreservation of all embrios}

Certainly the concept of cryopreservation of all embryos seems logical as a strategy to prevent OHSS given that OHSS is more common and more severe with pregnancy due to hCG-induced intrinsic ovarian stimulation.

Sills et al. in 2008 analyzed outcomes in patients undergoing elective embryo cryopreservation to prophylax against the development of OHSS (Sills ES et al., 2008).

Elective transfer of a single zona-free day 5 embryo and freezing of the supernumerary embryos or cryopreservation of all embryos for postponement of transfer can prevent the occurrence of late OHSS from pregnancy. However, it does not prevent early OHSS development due to exogenous hCG administration. The management based on elective 2 pronucleate embryo cryopreservation with subsequent thaw and grow-out to blastocyst stage for trasnsfer did not appear to compromise embryo viability or overall reproductive outcome.

The mean number of days from embryo cryopreservation to date of thaw embryo transfer was 115 (range 30-377), and embryo blastulation rate was $88 \%$. Finally, the live birth rate per embryo transfer was an excellent $43.6 \%$ in these patients. 
However, the Cochrane review found insufficient evidence in the literature to support the routine practice of embryo cryopreservation to prevent OHSS (D' Angelo A et al., 2002). As with all methods, it may reduce but not eliminate OHSS.

\subsection{Intravenous albumin at the time of oocyte retrieval}

Albumin is a low molecular weight plasma compound with a major impact on oncotic pressure. Human albumin has been used on the day of hCG administration in high-risk women to prevent OHSS. However, in a recent prospective randomized study, it was found that human albumin did not seem either to prevent or to reduce the incidence of severe OHSS. Without a concomitant reduction in vascular permeability, however, the effect of albumin on intravascular volume and hematocrit may be of short duration due to its diffusion into extravascular space, exacerbating both ascites and pleural effusions (Isikoglu M et al., 2007).

\subsection{Non-steroidal anti-inflammatory administration}

Low-dose aspirin therapy (100 mg daily, beginning on the first day of ovarian stimulation) was shown to be effective in preventing OHSS among high risk women in a recent study (Varnagy A et al., 2010). This study evaluated 2,425 cycles in which gonadotropin-releasing hormone agonist was used. Among 1,192 women at a high risk for developing OHSS, 780 randomly received aspirin, and the incidence of OHSS was $0.25 \%$ compared with $8.4 \%$ among the 412 women who did not receive aspirin. The pregnancy rates were similar.

\subsection{Dopamine agonist}

The most recently suggested strategy to prevent the development of OHSS is the use of dopamine agonists such as Cabergoline. The proposed mechanism is inhibition of phosphorylation of the VEGF receptor by Cabergoline, thereby preventing increased capillary permeability, the main action of VEGF.

In study by Alvarez et al. (2007), patients were assigned to receive either Cabergoline $0.5 \mathrm{mg} /$ day for 8 days starting on the day of hCG (35 patients) or placebo (32 patients). All patients underwent evaluation for OHSS, including serum hematocrit, ultrasound to evaluate for ascites, and magnetic resonance imaging (MRI) for ovarian venous permeability. They found that in the women who received Cabergoline, the ascites volume was statistically significantly lower than in those who received placebo. They also found that the percentage of women who developed moderate OHSS was statistically significantly lower in those patients who received Cabergoline. There was no difference in the percentage of patients in each group who developed severe OHSS.

In spite of these beneficial effects of the dopamine agonist cabergoline on vascular permeability without compromising implantation and pregnancy rates, this treatment would complement the ongoing progress with ither procedures such as in vitro maturation and oocyte vitrification and enable physicians to predict and prevent OHSS (Garcia-Velasco Ja et al., 2009). 


\subsection{In vitro maturation of oocytes (IVM)}

The safest way to prevent OHSS would be by not stimulating the ovaries. During an IVM cycle, immature oocytes are retrieved from barely stimulated or completely unstimulated ovaries. The oocytes are matured in defined culture media for $24-48 \mathrm{~h}$ and then fertilized by in vitro fertilization or intracytoplasmatic sperm injection. The embryo transfer is performed as usual; normally two embryos are transferred in two or three days after fertilization. The lack of ovarian stimulation during IVM cycles brings many benefits, including the following: reduction in medication cost, no risk for OHSS, and a reduction in the total number of patient visits for clinical and laboratory evaluations (Lazendorf SE et al., 2006).

Clinical trials evaluating IVM performed in women with PCOS demonstrated good pregnancy ratio per embryo transferred (20-54\%) and good implantation rates $(5.5-34.5 \%)$ (Suikkari aM et al., 2008). When evaluating IVM performed in ovulatory women, the results were a little worse, with pregnancy per embryo transfer rates between 15 and $33.3 \%$ and implantation rates between 8.8 and $22.6 \%$.

Although good results have been reported by some clinics, IVM has not yet become a mainstream fertility treatment. The most important reasons are: technical difficulties for retrieving immature oocytes from unstimulated ovaries and to cultivate them, lower chance of a live birth per treatment compared with conventional in vitro fertilization, the report of higher rates of meiotic spindle and chromosome abnormalities from immature human oocytes (Lazendorf SE et al., 2006).

\section{Management}

\subsection{Outpatient management}

Patients with mild manifestation of OHSS do not require any specific treatment. Outpatient surveillance is, nevertheless, mandatory to detect cases that may progress to moderate or severe OHSS. Most patients with moderate OHSS still can be managed on an outpatient basis, but they require more careful evaluation including daily weight and abdominal girth measurement, physical and ultrasound examination to detect increasing ascites, and to measure ovarian size. Oral fluid intake should be maintained at no less than $1 \mathrm{~L}$ per day. Women should be encouraged to drink to thirst, rather than to excess. Strenuous exercise and sexual intercourse should be avoided. Strict bed rest is unwarranted and may increase risk of thromboembolism. Progesterone supplementation is continued in the luteal phase, but supplementation is never done with hCG.

Discomfort may be relieved with acetaminophen or opiate medications if severe. Nonsteroidal anti-inflammatory agents are not recommended because they may compromise renal function in patients with OHSS.

Laboratory investigations that are helpful in assessing the severity of OHSS are haemoglobin,haematocrit, serum creatinine and electrolytes and liver function tests. Baseline values may help track the progress of the condition.

Review every 2-3 days is likely to be adequate. However, urgent clinical review is necessary if the woman develops increasing severity of pain, increasing abdominal distension, shortness of breath and a subjective impression of reduced urine output (Jenkins JM et al., 2006). 


\subsection{Hospital management}

Women with serious illness or severe OHSS require hospitalization for more careful monitoring and aggressive treatment. Careful and frequent reevaluation of the hospitalized patient with severe OHSS is essential. Clinical examination includes an assessment of hydration and cardiorespiratory system. Abdominal circumference and weight should be recorded at admission and daily until resolution. Fluid intake and output should be recorded and monitored on at least a daily basis. Urine output of less than 1,000 $\mathrm{mL} /$ day is a matter of concern.

Biochemical monitoring should include serum electrolytes, renal and liver function tests, a coagulation profile, and blood count. Sonographic examination provides assessment of ovarian size and the presence of ascites as well as pleural, or pericardial effusions. A chest X-ray and pulse oximetry are mandatory for any patient with respiratory symptoms and signs suggestive of hydrothorax, pulmonary infection, or pulmonary embolism. Assay of bhCG will help to diagnose pregnancy as early as possible. Pain and ascites can easily mask adnexal torsion, ovarian rupture, and acute intra-abdominal hemorrhage. Serial clinical and laboratory evaluations provide the means to monitor progression of illness and to recognize evidence of resolution (Jenkins et al., 2006).

Antiemetic drugs used should be those appropriate for the possibility of early pregnancy, such as prochlorperazine, metoclopramide and cyclizine.

The management of OHSS is essentially supportive until the condition resolves spontaneously.

Symptomatic relief is important, particularly regarding pain and nausea. Discomfort may be relieved with paracetamol or opiate medications if severe. If opiates are used, particularly in women with reduced mobility, care should be taken to avoid constipation. Nausea is usually related to the accumulation of ascites and so measures described to reduce abdominal distension should provide relief. Counselling support for both the woman and her partner provides reassurance and information to allay anxiety.

Regarding fluid management, it is critical to correct hemoconcentration. It is recommended to start with an initial IV fluid bolus of 500-1,000cc normal saline (NS) over the first hour of admission, followed by rehydration at a decreased rate of $30 \mathrm{cc} /$ hour using D5NS titrated to urine output. Oral fluid intake should be reduced to thirst and patient comfort. Albumin (25\%) may be needed for intravascular volume repletion, in which case $50-100 \mathrm{gm}$ of IV albumin is infused over 4 hours and repeated at 4-12 hour intervals as needed.

Hyperkalemia is treated in the standard manner with Kayexelate, insulin/glucose, sodium bicarbonate, or albuterol as needed. Rarely, diuretics can be given, but extreme caution is used in the administration of diuretics to patients with OHSS given their known intravascular volume depletion. Other inpatient management strategies include paracentesis if needed. Thromboembolism prevention is critical given the hypercoagulatory state of OHSS. It is generally recommended both venous support stockings and anticoagulants such as heparin 5,000 U subcutaneously twice daily. Occasionally, patients may need intensive care unit admission for thromboembolic complications, renal failure, or if invasive hemodynamic monitoring is indicated (central venous pressure, pulmonary capillary wedge pressure) (Alper MM et al., 2009). 


\section{References}

Alper MM, Smith LP, Sills ES. Ovarian hyperstimulation syndrome: current views on pathophysiology, risk factors, prevention, and management. J Exp Clin Assist Reprod. 2009 Jun 10;6:3.

Alvarez C, Marti-Bonmati L, Novella-Maestre E, Sanz R, Gomez R, Fernandez-Sanchez M, Simon C, Pellicer A. Dopamine Agonist Cabergoline Reduces Hemoconcentration and Ascites in Hyperstimulated Women Undergoing Assisted Reproduction. J Clin Endocrinol Metab 2007;92(8):2931-2937.

Binder H, Dittrich R, Einhaus F, Krieg J, Muller A, Strauss R, et al. Update on ovarian hyperstimulation syndrome: Part 1 -incidence and pathogenesis. Int J Fertil Womens Med 2007;52:11-26.

Chan WS. The 'ART' of thrombosis: a review of arterial and venous thrombosis in assisted reproductive technology. Curr Opin Obstet Gynecol 2009;21:207-18.

Chen SU, Chen RJ, Shieh JY, Chou CH, Lin CW, Lu HF, et al. Human chorionic gonadotropin up-regulates expression of myeloid cell leukemia-1 protein in human granulosa-lutein cells: implication of corpus luteum rescue and ovarian hyperstimulation syndrome. J Clin Endocrinol Metab 2010;95:3982-92.

D'Angelo A, Amso N. Embryo Freezing for Preventing Ovarian Hyperstimulation Syndrome. Cochrane Database Syst Rev 2002;2:CD002806.

Enskog A, Henriksson M, Unander M, Nilsson L, Brannstrom M. Prospective study of the clinical and laboratory parameters of patients in whom ovarian hyperstimulation syndrome developed during controlled ovarian hyperstimulation for in vitro fertilization. Fertil Steril 1999;71(5):808-814.

Fabregues F, Balasch J, Gines P, Manau D, Jimenez W, Arroyo V, et al. Ascites and liver test abnormalities during severe ovarian hyperstimulation syndrome. Am J Gastroenterol 1999;94:994-9.

García-Velasco JA, Isaza V, Quea G, Pellicer A. Coasting for the prevention of ovarian hyperstimulation syndrome: much ado about nothing? Fertil Steril. 2006 Mar;85(3):547-54.

Gracia-Velasco JA. Reprod Biomed Online. 2009;18 Suppl 2:71-5.

Golan A, Ron-El R, Herman A, Soffer Y, Weinraub Z, Caspi E. Ovarian hyperstimulation syndrome: an update review. Obstet Gynecol Surv 1989;44:430-40.

Goldsman MP, Pedram A, Dominguez CE, Ciuffardi I, Levin E, Asch RH. Increased capillary permeability induced by human follicular fluid: a hypothesis for an ovarian origin of the hyperstimulation syndrome. Fertil Steril 1995;63:268-72.

Griesinger G, Diedrich K, Devroey P, Kolibianakis EM. Gnrh Agonist for Triggering Final Oocyte Maturation in the GnRH Antagonist Ovarian Hyperstimulation Protocol: a Systematic Review and Meta-Analysis. Hum Reprod Update 2006;12(2):159-168.

Isikoglu M, Berkkanoglu M, Senturk Z, Ozgur K. Human albumin does not prevent ovarian hyperstimulation syndrome in assisted reproductive technology program: a prospective randomized placebo-controlled double blind study. Fertil Steril. 2007;88:982-5.

Jenkins JM, Drakeley AJ, Mathur RS. The management of ovarian hyperstimulation syndrome. Green-top Guideline 2006 September;(5):1-11.

Lanzendorf SE. Developmental potential of in vitro- and in vivomatured human oocytes collected from stimulated and unstimulated ovaries. Fertil Steril. 2006;85:836-7. 
Madill JJ, Mullen NB, Harrison BP. Ovarian hyperstimulation syndrome: a potentially fatal complication of early pregnancy. J Emerg Med 2008;35(3):283-6.

Manau D, Balasch J, Arroyo V, Jimenez W, Fabregues F, Casamitjana R, Creus M, et al. Circulatory dysfunction in asymptomatic in vitro fertilization patients. Relationship with hyperestrogenemia and activity of endogenous vasodilators. J Clin Endocrinol Metab 1998;83:1489-93.

Mathur RS, Akande AV, Keay SD, Hunt LP, Jenkins JM.Distinction between early and late ovarian hyperstimulation syndrome. Fertil Steril 2000;73:901-7.

Papanikolaou EG, Pozzobon C, Kolibianakis EM, Camus M, Tournaye H, Fatemi HM, et al. Incidence and prediction of ovarian hyperstimulation syndrome in women undergoing gonadotropin-releasing hormone antagonist in vitro fertilization cycles. Fertil Steril 2006;85:112-120.

Practice Committee of American Society for Reproductive Medicine. Ovarian hyperstimulation syndrome. Fertil Steril 2008;90:188-93.

Selgas R, Del Peso G, Bajo MA. Intra-abdominal hypertension favors ascites. Perit Dial Int 2010;30:156-7

Sills ES, McLoughlin LJ, Genton MG, Walsh DJ, Coull GD, Walsh AP. Ovarian Hyperstimulation Syndrome and Prophylactic Human Embryo Cryopreservation: Analysis of Reproductive Outcome Following Thawed Embryo Transfer. J Ovarian Res 2008;1(1):7.

Soares SR, Gomez R, Simon C, Garcia-Velasco JA, Pellicer A. Targeting the vascular endothelial growth factor system to prevent ovarian hyperstimulation syndrome. Hum Reprod Update 2008;14:321-333.

Suikkari AM. In-vitro maturation: its role in fertility treatment. Curr Opin Obstet Gynecol. 2008;20:242-8

Tollan A. Holst N, Forsdahl F, Fadnes HO, Oian P, Maltau JM. Transcapillary fluid dynamics during ovarian stimulation for in vitro fertilization. Am J Obstet Gynecol 1990;162:554-8.

Tozer AJ, Iles RK, Iammarrone E, Gillott CM, Al-Shawaf T, Grudzinskas JG. The Effects of "Coasting" on Follicular Fluid Concentrations of Vascular Endothelial Growth Factor in Women at Risk of Developing Ovarian Hyperstimulation Syndrome. Hum Reprod 2004;19(3):522-528.

Varnagy A, Bodis J, Manfai Z, Wilhelm F, Busznyak C, Koppan M: Low-dose aspirin therapy to prevent ovarian hyperstimulation syndrome. Fertil Steril. 2010;93(7):2281-4.

Villasante A, Pacheco A, Ruiz A, Pellicer A, Garcia-Velasco JA. Vascular endothelial cadherin regulates vascular permeability: Implications for ovarian hyperstimulation syndrome. J Clin Endocrinol Metab. 2007;92:314-21. 


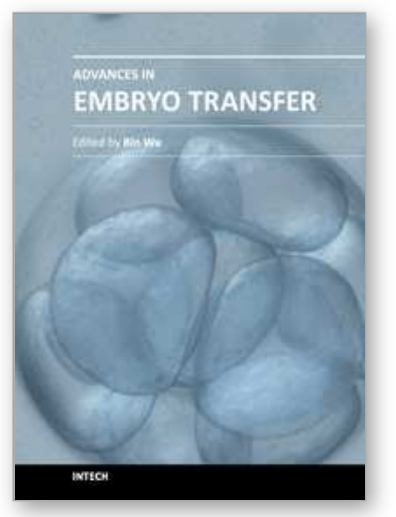

\author{
Advances in Embryo Transfer \\ Edited by Dr. Bin Wu
}

ISBN 978-953-51-0318-9

Hard cover, 248 pages

Publisher InTech

Published online 14, March, 2012

Published in print edition March, 2012

Embryo transfer has become one of the prominent high businesses worldwide. This book updates and reviews some new developed theories and technologies in the human embryo transfer and mainly focus on discussing some encountered problems during embryo transfer, which gives some examples how to improve pregnancy rate by innovated techniques so that readers, especially embryologists and physicians for human IVF programs, may acquire some new and usable information as well as some key practice techniques. Major contents include the optimal stimulation scheme for ovaries, advance in insemination technology, improved embryo transfer technology and endometrial receptivity and embryo implantation mechanism. Thus, this book will greatly add new information for readers to improve human embryo transfer pregnancy rate.

\title{
How to reference
}

In order to correctly reference this scholarly work, feel free to copy and paste the following:

Ivan Grbavac, Dejan Ljiljak and Krunoslav Kuna (2012). Prevention and Treatment of Ovarian Hyperstimulation Syndrome, Advances in Embryo Transfer, Dr. Bin Wu (Ed.), ISBN: 978-953-51-0318-9, InTech, Available from: http://www.intechopen.com/books/advances-in-embryo-transfer/prevention-andtreatment-of-ovarian-hyperstimulation-syndrome

\section{INTECH}

open science | open minds

\section{InTech Europe}

University Campus STeP Ri

Slavka Krautzeka 83/A

51000 Rijeka, Croatia

Phone: +385 (51) 770447

Fax: +385 (51) 686166

www.intechopen.com

\section{InTech China}

Unit 405, Office Block, Hotel Equatorial Shanghai

No.65, Yan An Road (West), Shanghai, 200040, China

中国上海市延安西路65号上海国际贵都大饭店办公楼 405 单元

Phone: +86-21-62489820

Fax: +86-21-62489821 
(C) 2012 The Author(s). Licensee IntechOpen. This is an open access article distributed under the terms of the Creative Commons Attribution 3.0 License, which permits unrestricted use, distribution, and reproduction in any medium, provided the original work is properly cited. 